Rev. Elev. Méd. Vét. Pays trap., 1964, 17, 4, (619-36).

\title{
Description de Rhipicephalus muhsamae n. sp. de l'Ouest-Africain (groupe de Rh. simus; Acariens, Ixodoidea)
}

\author{
par P. C. MOREL et G. VASSILIADES
}

\begin{abstract}
RÉSUMÉ
Rhipicephalus muhsamae n. sp, est notamment distinct de Rh. simus, du point de vue de sa morphologie, par la siructure du gonopore femelle; de Rh. senegolensis principalement par le gonopore femelle ef les plaques adanales des mâles. Rh. simus est distribué d'une façon typıque en Afrique orientale el australe ; Rh. muhsomae le remplace en Afrique occidentale à l'ovest du $\mathrm{Nit}$ et dans le bassin du Congo, jusqu'au Sénégal ; il est associé aux savanes tropicales et subtropicales, parfois aux subéquatoriales, ainsi qu'aux steppes sudsahéliennes, alors que Rh. senegolensis, plus hygrophile, habite normalement les savanes subéquatoriales, moıns souvent les subtropicales; dans l'OuestAfricain les distributions des deux espèces sont parallèles aux zones de végétation, où elles se remplacent mutuellement en fonclion des différences climatiques générales ou locales.
\end{abstract}

\section{DESCRIPTION}

Holotype : une femelle sur bøuf de Sangalkam (Rufisque, Sénégal) (15. V. 62).

Allotype : un mâle, mêmes données que cidessus.

Spécimens examınés : tous ceux marqués d'un astérisque dans la liste des localités de distribution.

Le nom de la nouvelle espèce a été choisi en hommage à Maie B. Feldman-Muhsam, pour l'importance de ses travaux sur !'utilisation du gonopore des femelles dans la systématique des Hyalomma et Rhipicephalus, et pour l'aide apportée au début de nos études sur ce dernier genre.

\section{Mâle (fig. 1 et 2)}

Capitulum - basis capituli environ 2 fois plus large que longue en vue dorsale; auricules au niveau du quart antérieur de la longueur de la basis (mesurée entre le niveau de la base interne des palpes et le bord postéro-dorsal de la basis) ; cornes basidorsales moyennes, en cône arrondi, saillantes par rapport au bord postérieur légèrement concave de la basis; peigne ventro-palpal à soies frangées; longueur du capitulum en vue dorsale (entre le niveau antérieur des palpes et le bord postérieur de la basis) : 0,70-0,90 $\mathrm{mm}$; largeur entre les aurıcules: 0,80-1,10 mm.

Face dorsale $\rightarrow$ conscutum à sillons scapulaıres à carènes courtes et marqués de grosses ponctuations non contiguës (5-8) ; sillons cervicaux courts ; sıllons marginaux complets délimitant sur le plus grand nombre des spécimens une seule paire de festons, à carène bien marquée et à grosses ponctuations en séries plus ou moins contigues dans la rainure; sillons médian et paramédians étroits et superficiels, à fond strié sans ponctuations, les paramédians arqués ef plus courts que le médian rectılıgne; ponctuations sétifères grosses, en séries bien visibles; 


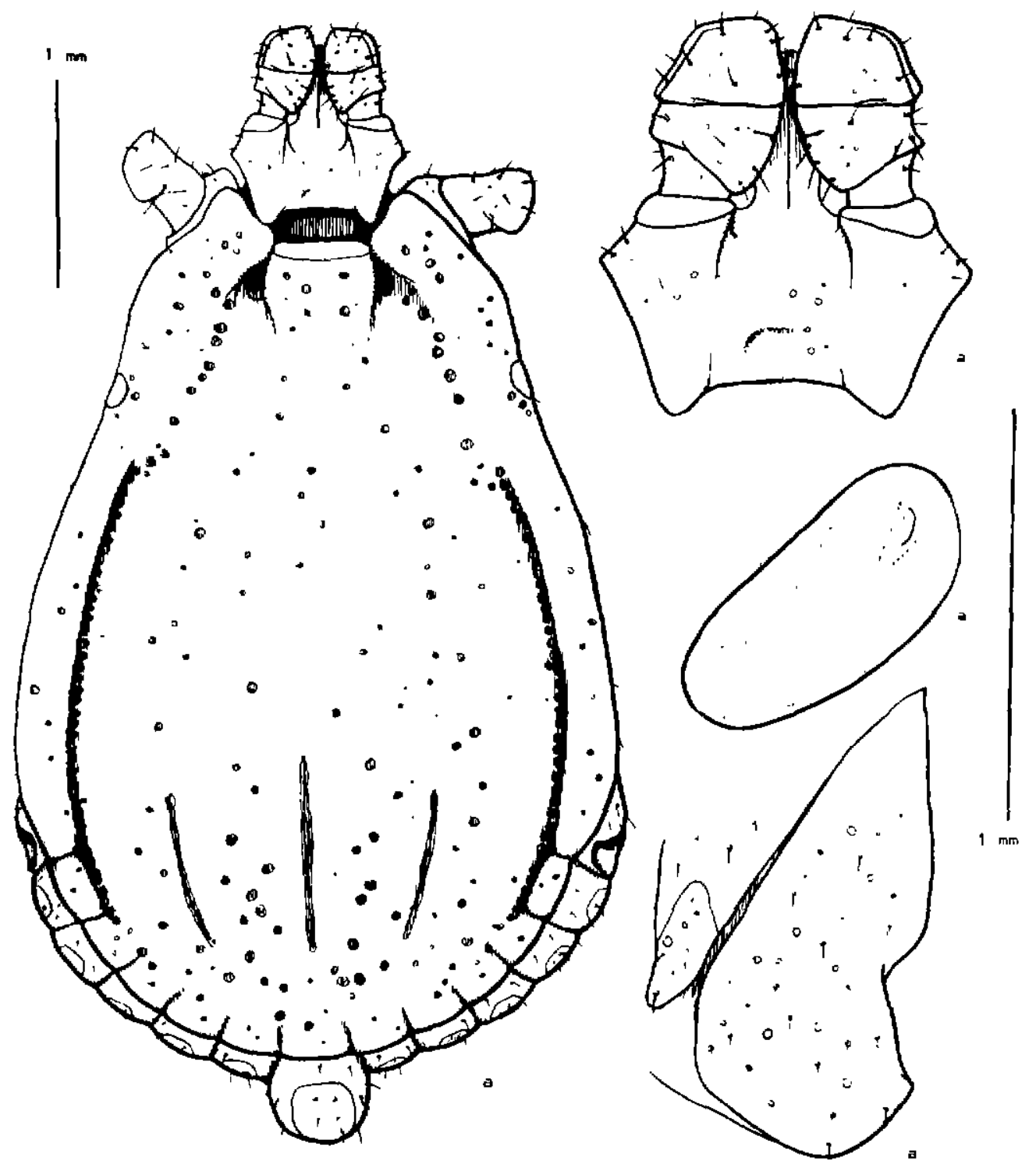

Fig. 1. - Rhipicephalus muhsomee, mâle: face dorsale; détails du capıtulum, du stigmate, des plaques ventrales (exemplaire de Sangalkam). 


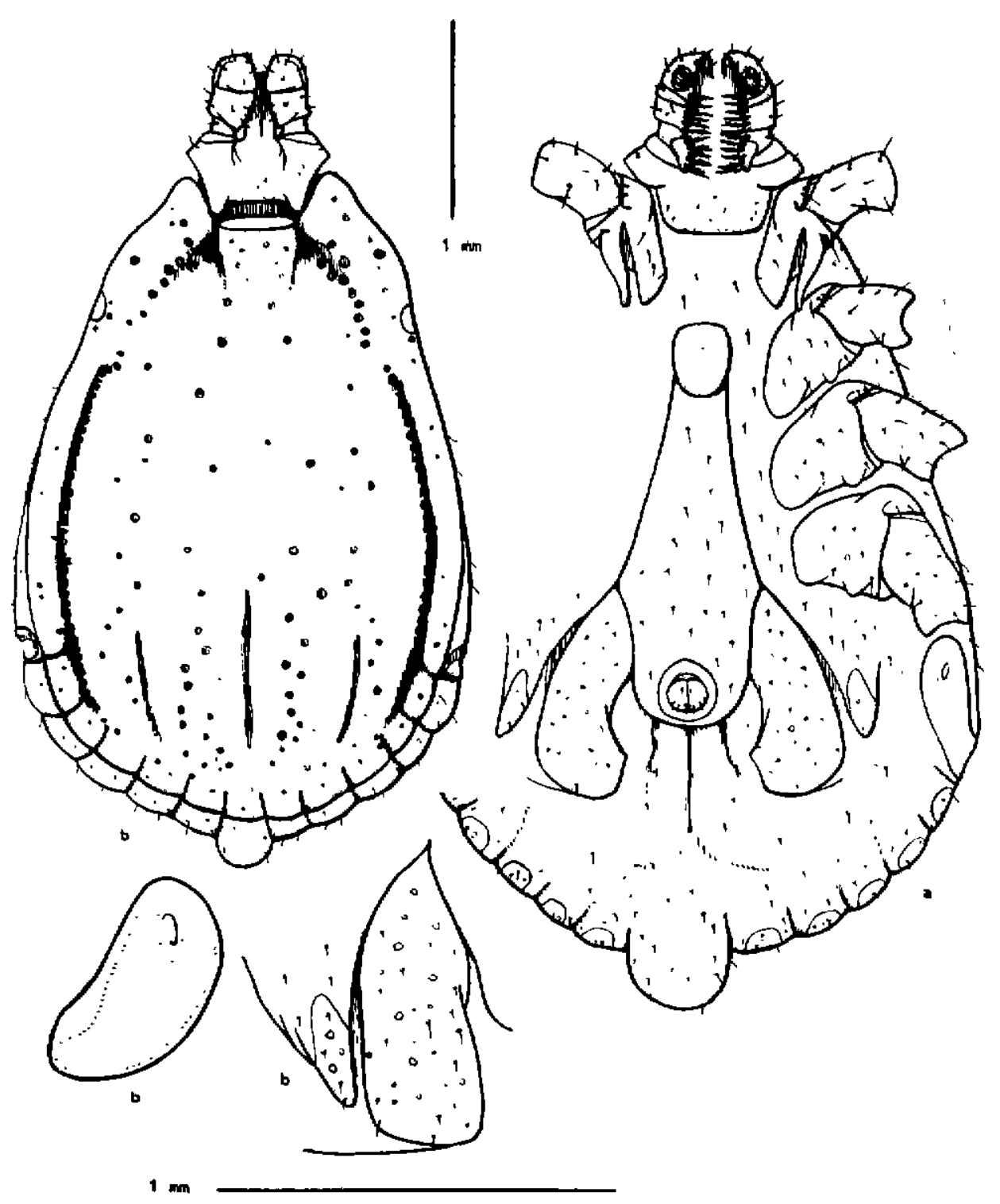

Fig. 2 - Rhipicephalus muhsamae, mâle ; face dorsale; détails du stigmate et des plaques ventrales (exemplaire de Sangalkam); face ventrale (exemplaire en vue dorsale de la fig. 1). 
ponctuations interstitielles peu nombreuses, éparses, fines ou très fines, donnant à l'ensemble du conscutum un aspect plutôt lisse ; longueur du conscutum : $3,10-4,40 \mathrm{~mm}$; largeur maximale du conscutum : 2,10-3,00 mm; chitine brunrouge ou brun-noir.

Face ventrale - coxa I à épine interne en lame allongée, à épine externe longue à pointe arrondie ; espace entre les épines en fente étroite courbe ; processus coxal I antérieurement visible en vue dorsale en avant des scapulae ; coxae IIIII-IV à épine interne en écaille large arrondie, à épine externe en pointe triangulaire plus ou moins mousse; stigmates réniformes ou en ovale allongé, à processus dorsal apparent sur la lame criblée.

Plaques ventrales - plaques adanales en bat- toir à légère concavité interne, à angle médiointerne obtus; plaques accessoires en pointe triangulaire.

Festons - chez les mâles gorgés, un seul feston saillant, le médian.

\section{Femelle (fig. 3 et 4 ).}

Capitulum - basis capituli environ 2 fois $1 / 2$ plus large que longue en vue dorsale; auricules au, niveau du tiers antérieur de la longueur de la basis (longueur mesurée entre le niveau de la base interne des palpes et le bord postérodorsal de la basis) : aires poreuses petites, circulaires ou ovalaires, distantes entre elles de 2 fois leur largeur, postérieures aù, niveau des

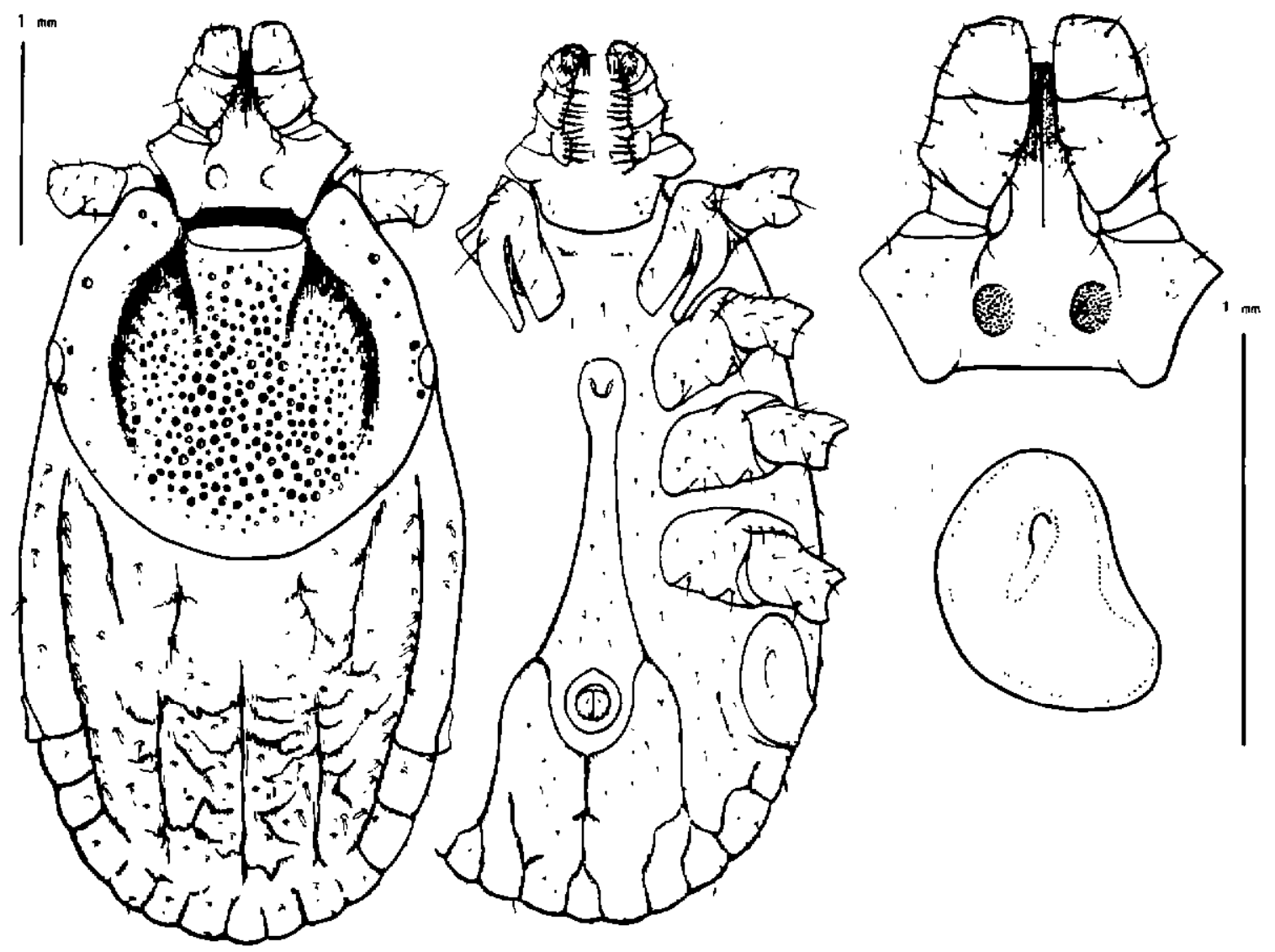

Fig. 3. - Rhipicephalus muhsomae, femelle; faces dorsale et ventrale; détails du stigmate et du capituium (exemplaire de Sangalkam). 
auricules; cornes basidorsales courtes, arrondies, saillantes par rapport au bord postérieur légèrement concave de la basıs: palpes trapus, 2 fois $1 / 2$ plus longs que larges; peigne ventropalpal à soies frangées; longueur du capitulum en vue dorsale (entre le niveau antérieur des palpes et ie bord postérieur de la basıs) : 0,60$0,90 \mathrm{~mm}$; largeur entre les auricules : $0,75-$ $1,10 \mathrm{~mm}$.

Face dorsale - scutum approximativement aussi large que long (longueur vraie, du niveau antérieur des scapulae au bord postérieur du scutum) ; longueur : 1,50-2,00 mm ; largeur : $1,70-2,10 \mathrm{~mm}$; sillons scapulaires complets, à carène et à série de grosses ponctuations dans la raınure, plus ou moins contiguës (8-12); sillons cervicaux moyens, ponctuations sétifères grosses, bien apparentes; ponctuations interstifielles fines ou très fines, peu nombreuses, éparses, donnant à l'ensemble du tégument un aspect plutôt luisant; alloscutum à soies bacilliformes.

Face ventrale - coxae de morphologie analogue à celle des mâles; stigmates ovoides, à processus dorsal marqué sur la lame criblée.

Gonopore femelle - lèvre trapézolde à bord postérieur rectiligne ou concave, à bords latéraux convexes, à rebords très larges en bourrelets hyalins: sclérites de l'atrium drots ou légèrement courbes, à concavité externe.
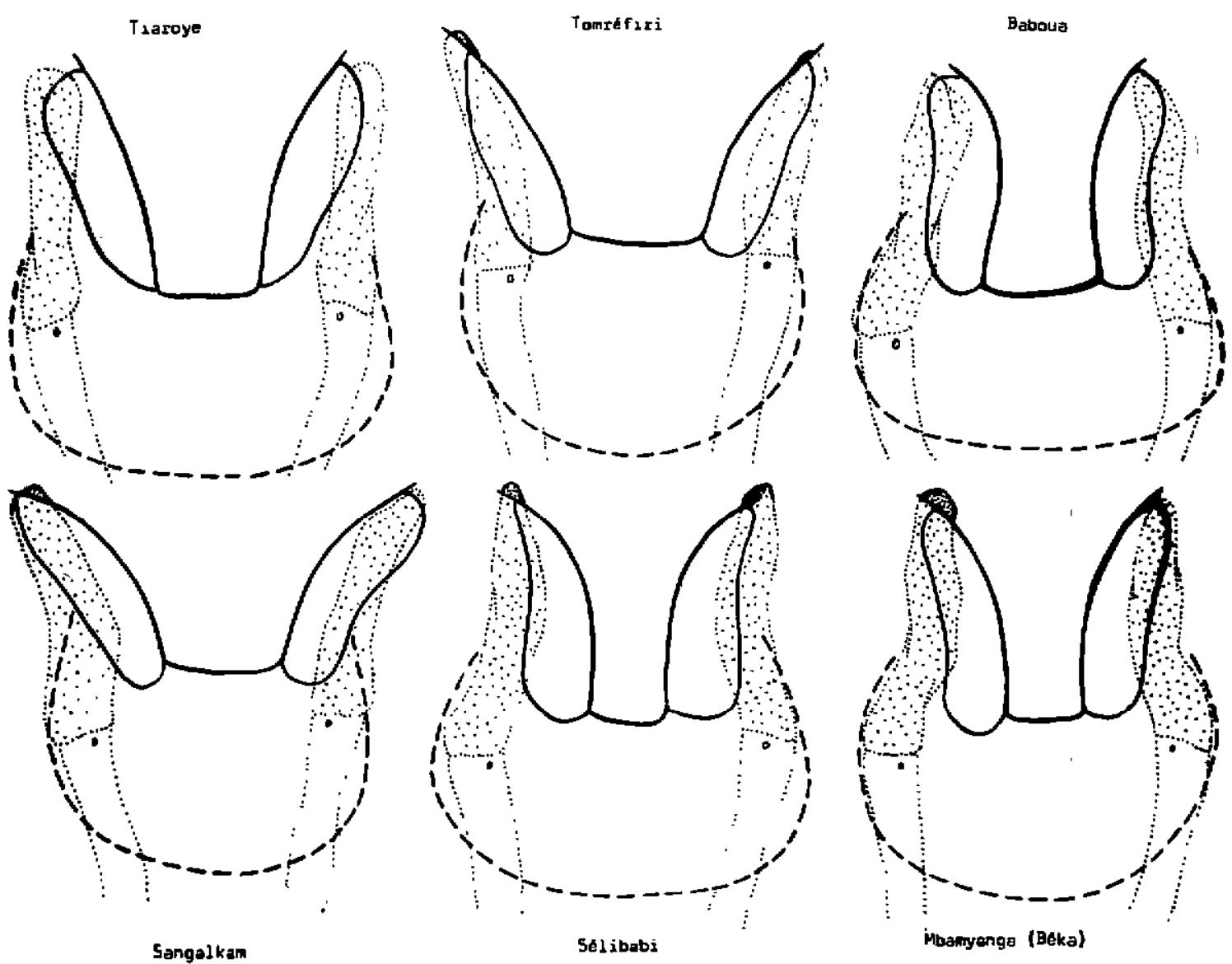

Fig. 4. - Rhiprcephalus muhsamoe, femelle; détall du gonopore (exemplaires de Sangalkam, Tiaroye, Tomréfiri, Sélibabi, Baboua, Béka). 


\section{COMPARAISONS \\ AVEC LES ESPÈCES VOISINES}

Mâles (Rh. senegalensis : fig. $5-7 ;$ Rh. sımus : fig. 11-12).

Par ses plaques adanales, Rh. muhsamae se rapproche seulement de $R$ h. simus ; la morphologie des mâles des deux espèces est extrêmement analogue et la diagnose en est difficile ; peut-être la courbure du processus dorsal du stigmate, telle qu'elle est dessinée par la lame criblée, fournirait-elle un critère de différenclatıon utilisable, à employer sur un certain nombre d'exemplaires, comme toutes les fois qu'on a recours aux caractères des stigmates (cf. leur application dans le groupe de $R h$. songuineus) ; chez Rh. simus le processus dorsal se détache presque perpendiculairement de la lame criblée, tout au moins sur son bord externe : chez $R h$. muhsamae, ce processus se détache selon une courbe large correspondant à un angle obtus

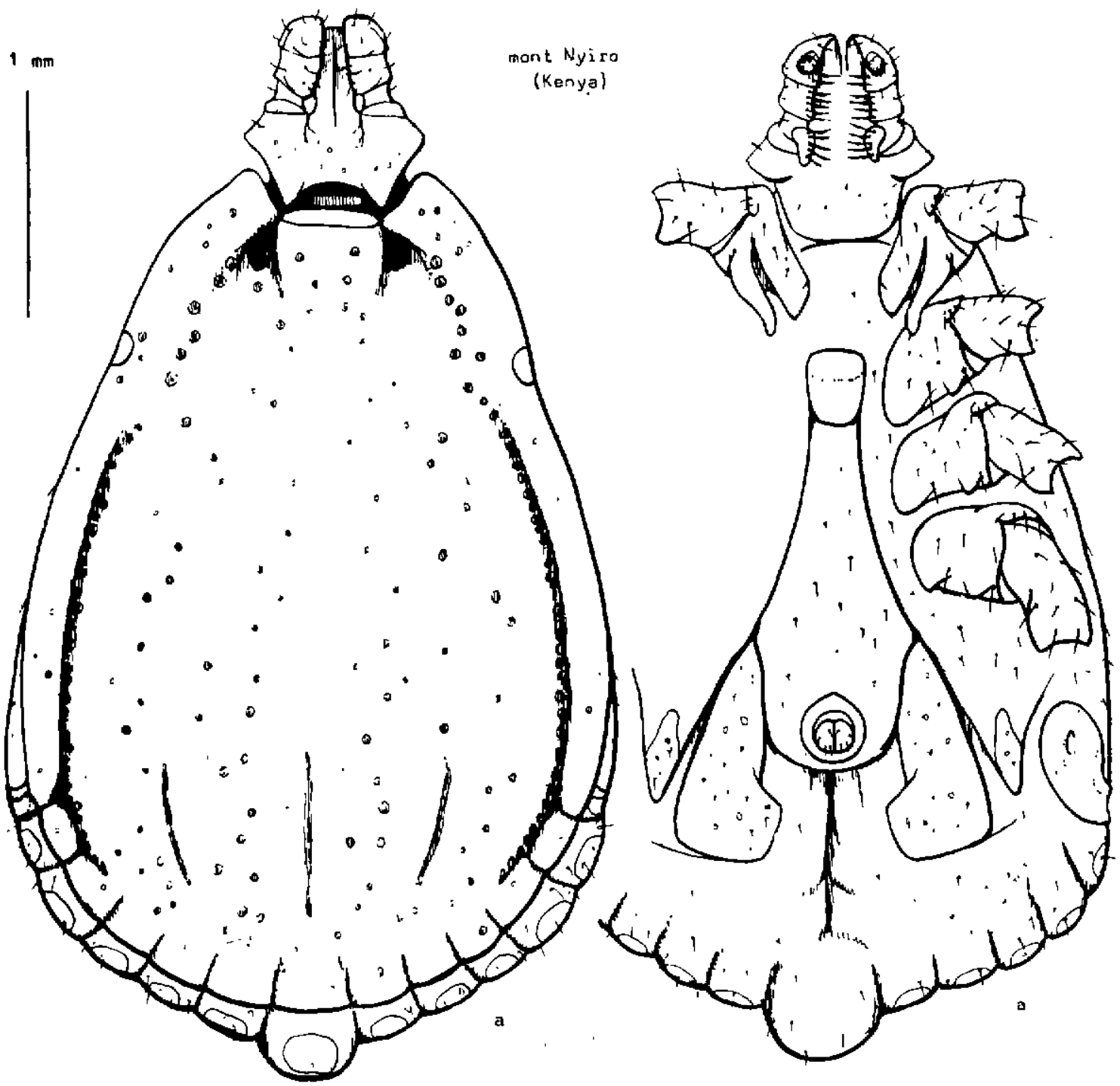

Fig. 5. - Rhipicephalus simus, mâle ; faces dorsale et ventrale (exemplaire du mont Nyiro). 
très ouvert; la diagnose dolt toujours être confirmée sur les femelles; la distribution géographique permet par la suite une généralisation plus rapide, sauf dans le bassin du Nil ( $c$. la distribution). Les plaques adanales de $R h$. senegalensis d'une part, en faucilles, celles de Rh. lunulatus d'autre part, à concovité postérieure et éperon postéro-extenre, permettent de distinguer aisément ces espèces.

Femelles (Rh. senegalensis: fig.8-10; Rh. simus: fig. 13-14).

Les rebords latéraux du gonopore en bourrelets hyalins permettent de distınguer d'emblée Rh. muhsamae; la lèvre elle-même est de forme trapézolde; chez Rh. simus, Rh. senegalensis,
$R h$. iunulatus, les lèves sont en $U$ ou en $V$ plus ou moins arrond, à rebords minces ou nuls; les sclérites de l'atrium sont analogues chez $R h$. muhsomae ef $R h$. simus; chez $R h$. senegalensis ils sont plus allongés et concaves extérieurement ; chez Rh. lunulatus au contraire, les sclérites sont assez courts, distants (lèvre en largeur) et convexes extérieurement.

En ce qui concerne les sillons du scutum, Rh. simus présente une carène scapulaire très courte, et le sillon correspondant n'est représenté que par les ponctuations grosses, alignées dans la rainure scapulaire et non contigues; il en va de même pour Rh. lunulatus; chez Rh. senegalensis, le sillon est complet, à corène aussi longue que la rainure.

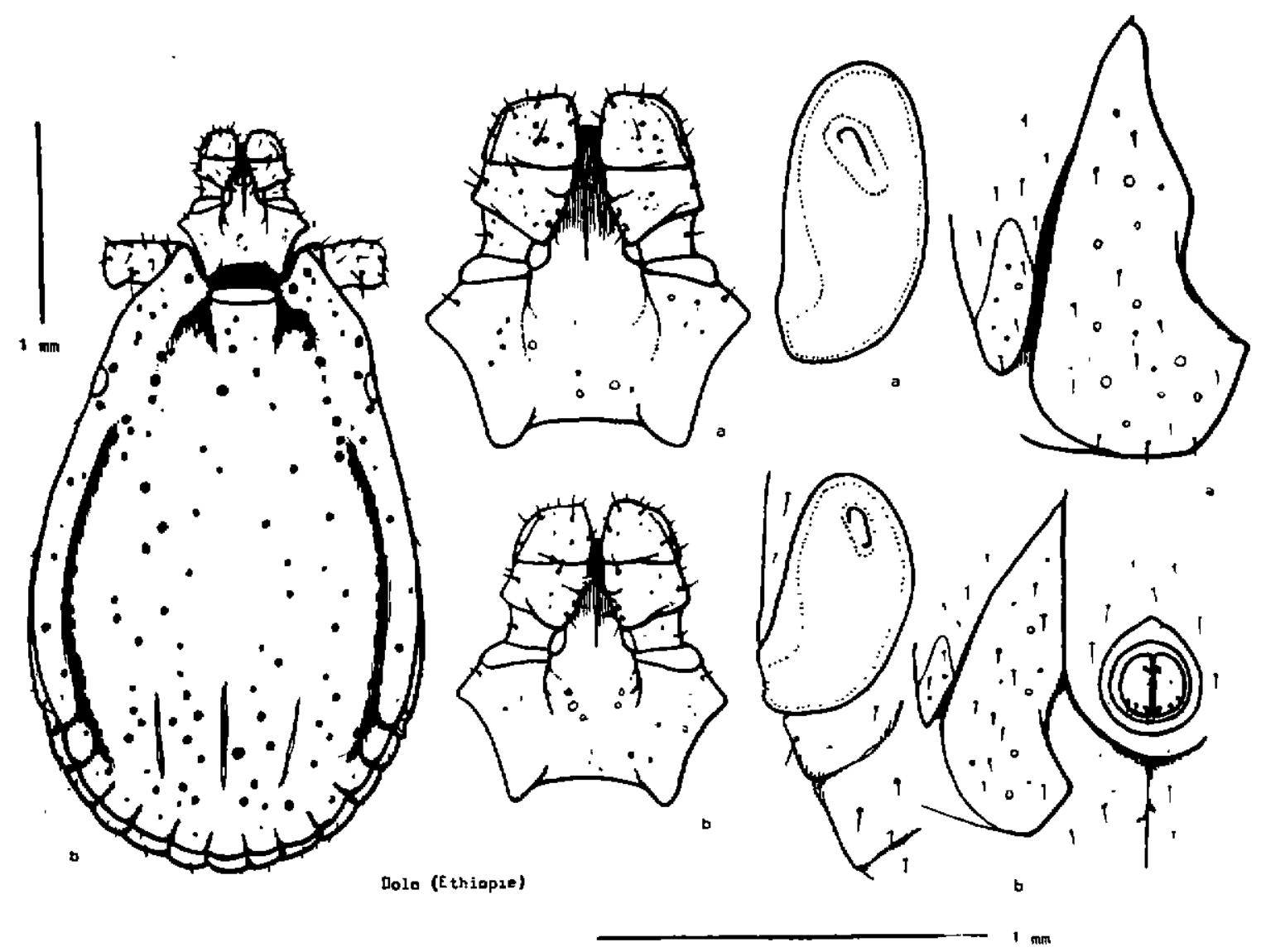

Fig. 6. - Rhipicephaius simus, mâle ; face dorsale ; détails du capitulum, du słıgmate el des plaques ventrales (exemplaire de Dolo); détails du capitulum, du stigmate et des plaques ventrales (mâle de la fig. 5). 


\section{DISCUSSION}

Le gonopore femelle de $R h$. muhsamae a été clairement décrit et représenté par TENDEIRO $(1959,21: 37-47$; fig. 8, photographies 5 et 6$)$, malheureusement sous le nom de $R h$. senegalensis, à la suite de confusions sur la nature spécifique de la population des Rhipicephalus du groupe de $R h$. simus qu'il avait rencontrée en Guiné-Bissau.

Les syntypes de Rh. erlangeri Neumann, 1902 (1 ${ }^{*}$, coll. Neumann no 1152, Daroli, Ethiopie: cheval), Rh. hilgerti (1 $\approx$ \& 1 \&, coll. Neumann no 1153 ; Daroli : Canis voriegatus), Rh. shipleyi Neumann, 1902 (1 o 1ㅇ, coll. Neumann no 1150; Sudan : hyène), personnellement examinés, appartiennent à l'espèce Rh. simus, notam- ment les femelles de Rh. hilgerti ef Rh. shipleyl; par son origine, ce dernier nom pouvait risquer de s'appliquer à l'éspèce rencontrée du Sénégal au Tchad; il n'en est rien ; il est à supposer que les exemplaires provenaient de la rive orientale du Nil, des confins de l'Ethiopie ou de l'Uganda.

\section{DISTRIBUTION DE RHIPICEPHALUS MUHSAMAE}

Les exemplaires ou les séries personnellement observés, qui ont fait l'objet de publications ou cités ici pour la première fois, sont signalés d'un astérisque.

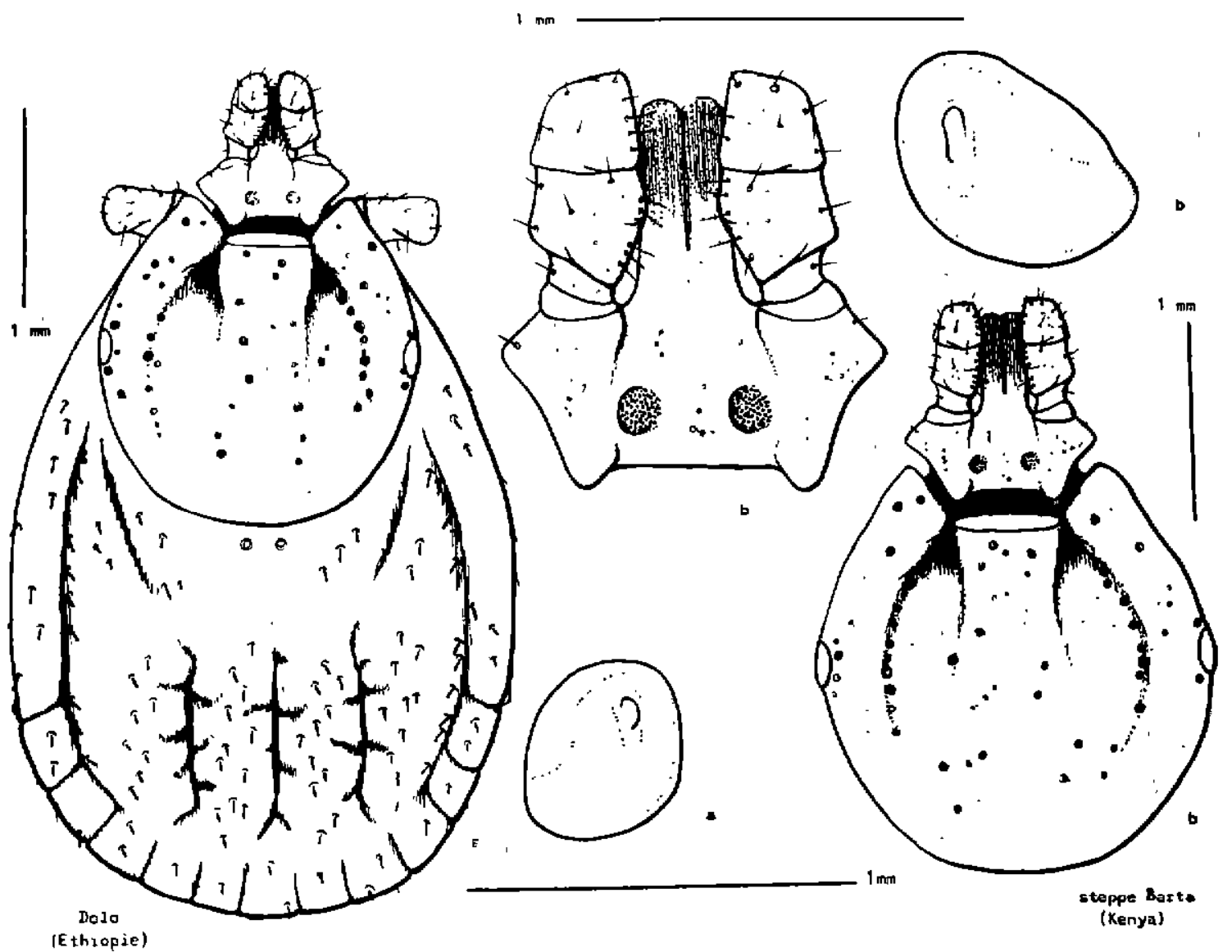

Fig. 7. - Rhipicephalus simus, femelle; face dorsale et stıgmate (exemplaire de Dolo) ; capitulum, scutum et stigmate (exemplaire de Barta). 


\section{Guine-Bissau}

TENDEIRO (1946, 397, Rh. simus) : Pessuba (Bissau). TENDEIRO $(1948,639$, Rh. simus) : Bissau ; Bissora ; Conchungo. TENDEIRO (1951, 909, Rh. s. simus) : Bor (Bissau) ; Mansoa ; Pessuba (Bissau). TENDEIRO (1959, 21 : 37, Rh. senegolensis) : Farim, Bissorâ ; Bissau.

\section{Haute-Volta}

*MOREL (1958, 153, Rh. s. simus) : Bobo-Dioulasso: Samandéni (Bobo); Banankélédaga (Bobo) ; Santidougou (Bobo) ; Tiéfora (Banfora).

* références nouvelles : Badéma (Bobo); Dou- goumato; Koumbia (Houndé),; Kokologo (Koudougou).

Mali

ROUSSELOT $(1951,307 ; 1953: 40$ et $89 ;$ Rh. simus) : Bamako; Ségou.

*MOREL (1958, 153, Rh. s. simus) : Lorakbane (Nioro) ; Sokolo; Ténenkou (Massina); Sansa (Gniminiama); Bamako : Sotuba (Bamako); San.

LAMONTELLERIE $(1960,750$, Rh. simus) : Diou (Sikasso); Fingolo (Bougoula).

*références nouvelles : Douentza; Famana (Bougouni); Yanfolila (Bougouni) ; Gao.

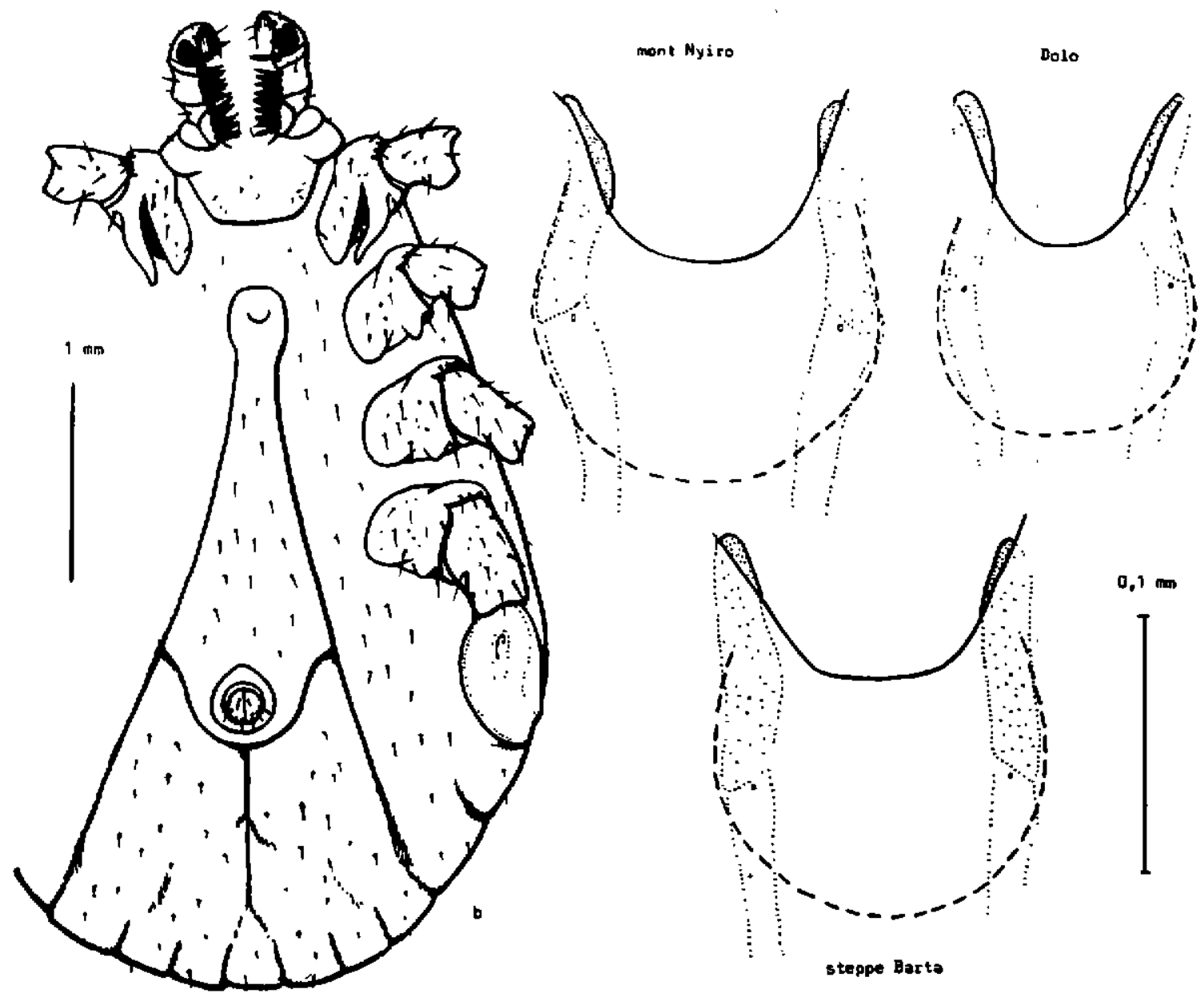

Fig. 8. - Rhipicepholus simus, femelle; face ventrale (exemplaıre de Barta) ; délail du gonopore (exemplaires de Dolo, Barta, du mont Nyiro). 
Mauritanie

*références originales : Bafréchié (lac Rkiz); Sélibabi.

\section{Niger}

*référence originale : Niamey.

Nigeria

UNSWORTH (1952, 331, Rh. s. simus) : Numan,

Shellen, Tungo, Uba (Adamawa); Bauchi,
Gombe, Misau, Zungor (Bauchi Pr.) ; Auno, Bama, Biu, Maidugari, Potiskum (Bornu); Ilorin ; Kano; Dawdawa, Funtua, Katsina, Tambu (Katsina Pr.) ; Abuja, Bida, Kontagora (Niger) ; Anchau (Zaria).

\section{Sénégal}

*MOREL (1956, $229 ; 1958,153 ;$ Rh. s, simus ; 1961, 83, Rh. simus) : Niokolo-Koba; Badi.

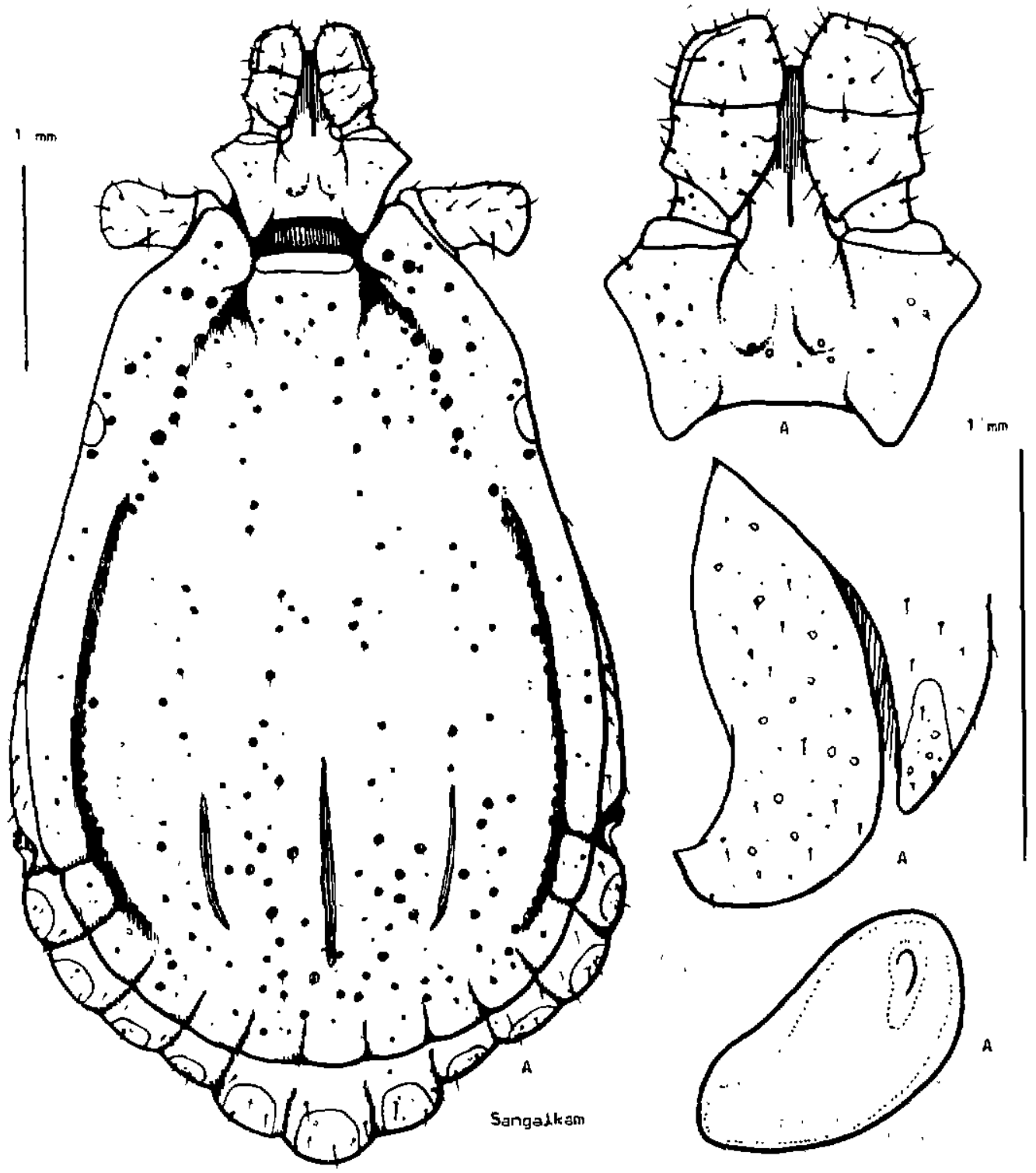

Fig. 9. - Rhipicephalus senegolensis, mâle; face dorsale, détaıls du capilulum, du stigmate et des plaques ventrales (exemplaire de Sangalkam). 
*MOREL (1958, 153, Rh. s. simus) : Sangalkam (Rufisque); Thiès ; Diassane (Thiès) ; Niakhar; Souli (Bakel); Nayes; Nganda (Kaolak): Hamdallaye (Birkelane); Tambakounda ; Kédougou ; Sédiou.

*MOREL $(1958,153$, Rh. simus senegalensis) : Keur Bouki (Kaolak); Mbayène (Koungheul); Ziguinchor. *références nouvelles : Tioroye (Dakar) ; Pout (Sébikhotane); Mbour; lac de Guiers; Sangalkam; Kolda ; Ziguinchor.

\section{Congo-Ouest}

*références personnelles: Cabinda, Brazzavilie : Pointe-Noire.

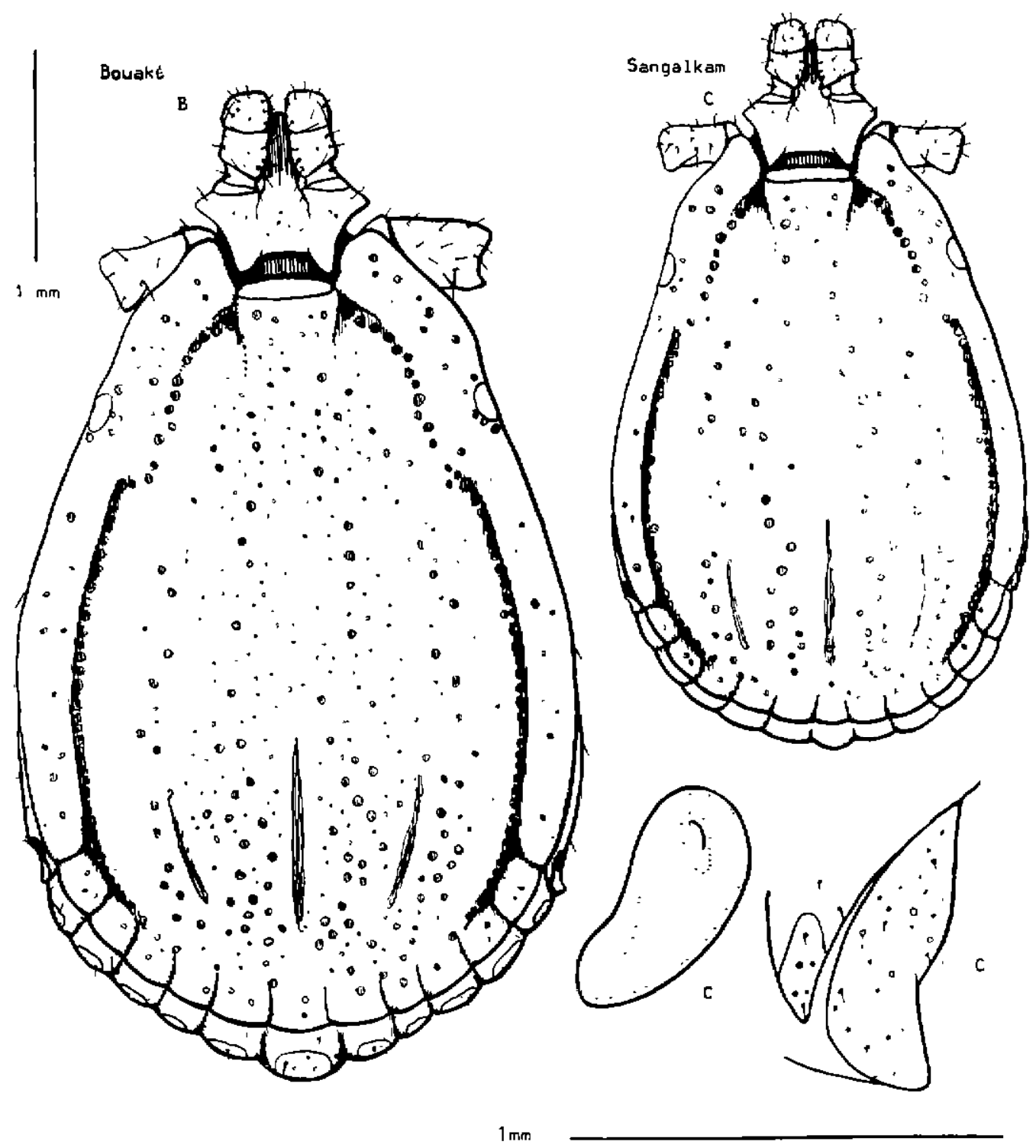

Fig. 10. - Rhipicephalus senegalensıs, mâle; face dorsule (exemplaire de Bovaké); face dorsale : dérails du śligimale et des plaques ventrales (autre exemplaıre de Sangalkam). 


\section{Cameroun}

ZUMPT (1943, 1-24, Rh. simus) : Garoua.

* MOREL \& MOUCHET (1958, 69, Rh. s. simus) : Mbamyanga (Béka); Djamba.

* MOREL \& MAGIMEL (1959, 53, Rh. s. simus) :

Fort-Foureau; Goulpei, Brigadou (Fort-

Foureau); Riggıl (Fort-Foureau).

*MOREL \& GRABER (1961, 199, Rh. simus) :

Tchévi, Chaova (Fort-Foureau),

* MOREL \& MOUCHET (1964, sou; presse) :

Fort-Foureau ; Logone-Bırni ; Maroua ; Petyé

(Maroua); Wasa.

\section{Centre-Afrique}

ROUSSELOT $(1951,307 ; 1953: 40$ et $89 ;$ Rh. simus) : Bangui.
*MOREL \& FINELLE (1961, 191, Rh. simus) : Besson (Babova) ; Fort-Sibutt.

*références nouvelles : Batangafo; Bambari.

\section{Congo-Equateur}

? THEILER \& ROBINSON (1954, 447, Rh, simus) :

Coquilhatville; Yanangu.

\section{Congo-Kwango}

? NEWSTEAD, DUTTON \& TODD (1907, 3-112, Rh. simus) : Banana.

? ROUBAUD \& VAN SACEGHEM (1916, 763, Rh. simus) : Zambi.

? SCHWETZ (1927, 81, Rh. simus) : Bolobo ; Kunungu.

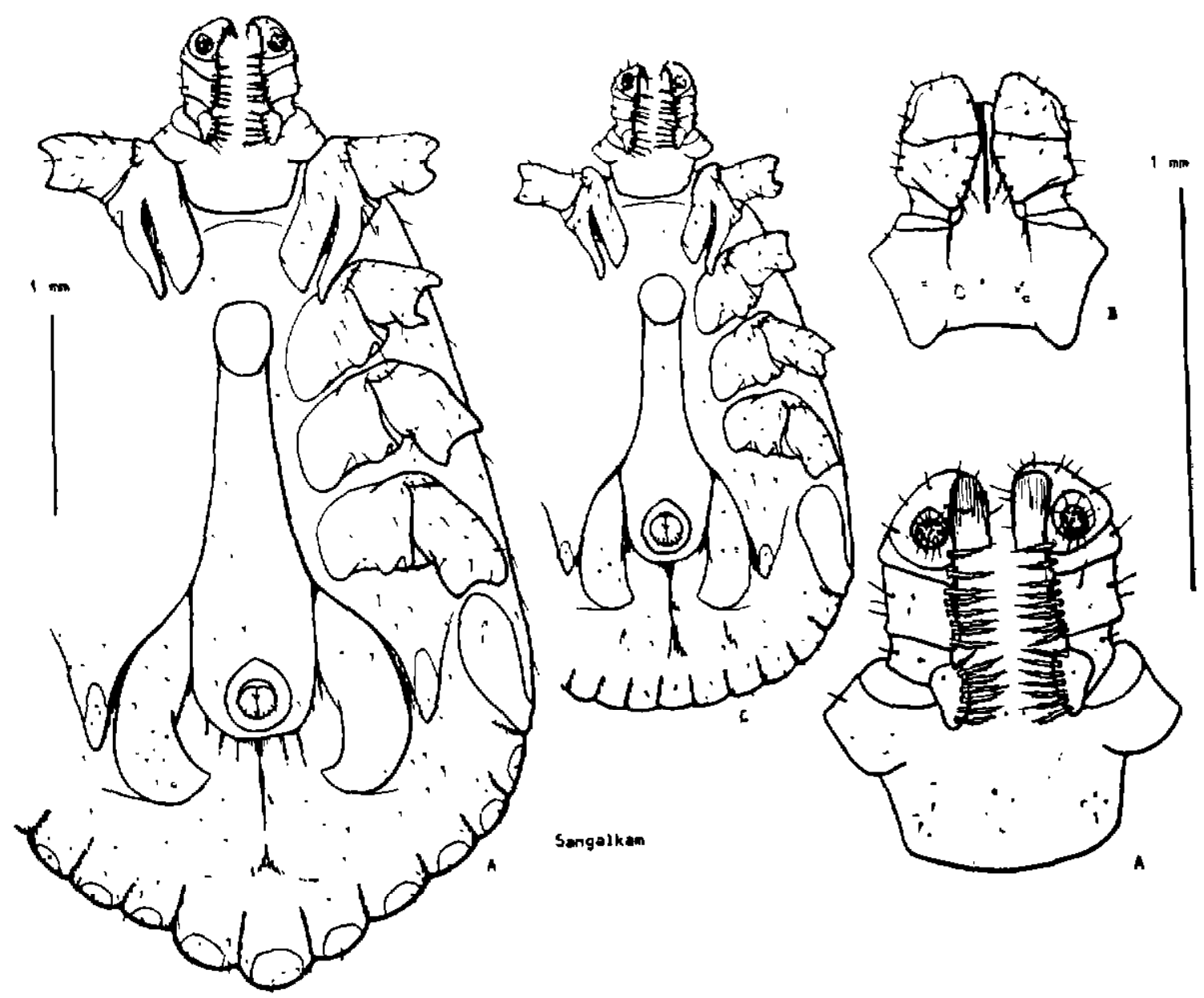

Fig. 11. - Rhipicephalus senegolensis, mâle ; faces ventrales; détails du capitulum, face ventrale et dorsale (exemplaires de Sangalkam). 
? BEQUAERT (1931, 209, Rh. simus) : Banana ; Bolobo; Kunungu.

? THEILER \& ROBINSON (1954, 447, Rh. sImus) : Banana ; Léopoldville.

\section{Congo-Oriental}

TONELLI-RONDELLI (1930, 112, Rh. simus); ZUMPT (1943, 1-24, Rh. simus) : Aba. BEQUAERT (1931, 209, Rh. simus) : Medje. SCHWETZ (1932, 549, Rh. simus): Gwane.

THEILER \& ROBINSON $(1954,447$, Rh. simus): Gwane ; Medje ; Aru.

VAN VAERENBERGH $(1954,222$, Rh. simus) : Bunia.

*référence personnelle : Gwane (coll. Schwetz).

\section{Sudan}

? HOOGSTRAAL (1956: 732-733, Rh. s. simus) : [Bahr el Ghazal] Galual-Nyang forest : Jur river ; Lau ; Fanjak; Wau ; Kenisia: Yirol. [Darfur] Zalingei ; Kulme. [Kordofan] Tabanga: Talodi. [supposition en fonction de la biogéographie].

? HOOGSTRAAL \& THEILER $(1959,217$, Rh. $s$. simus) : Galual-Nyang forest.

*MOREL \& MAGIMEL (1959, 53, Rh. s. simus) : Fort-Lamy ; Tomrefiri (Fort-Lamy).

\section{Tchad}

*MOREL \& GRABER (1961, 199, Rh. simus): Bol ; Am Siléna ; Iriba; Abéché ; Dai ; FortArchambault.
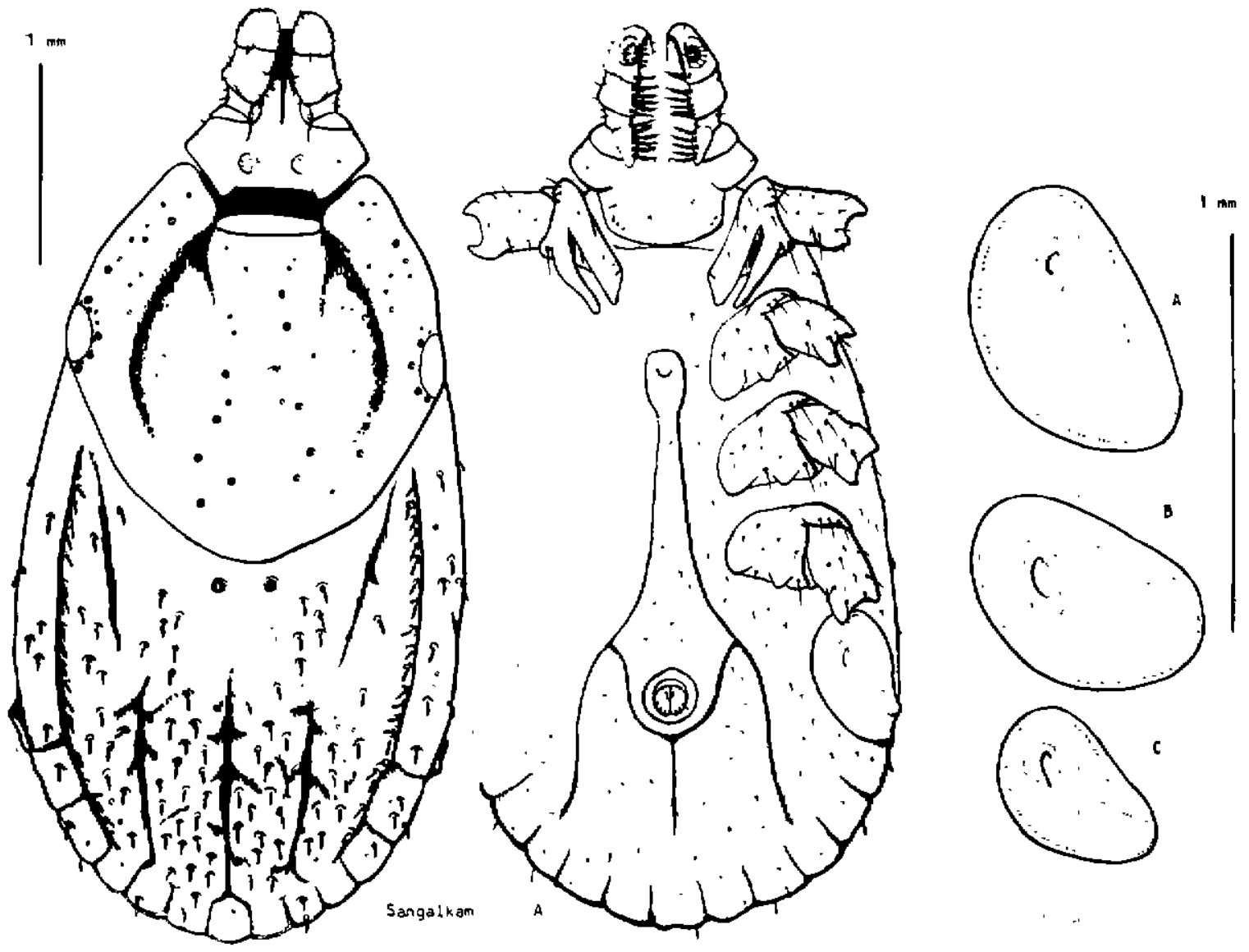

Fig. 12 - Rhipicephalus senegalensis, femelle; face dorsale; détails de stigmates (exemplaires de Sangalkam). 


\section{HABITAT}

L'habitat normal de l'espèce semble les savanes tropicales soudaniennes, où sa distribution semble régulière et où ne se rencontrent pas normalement $R h$. senegalensis et $R h$. simus; il en va de même pour les steppes xérophytes sudsahéliennes, oủ cependant $R h$. muhsamoe est restreint aux formations denses des abords des cours d'eau et lacs. Dans les savanes subtropicales sud-soudaniennes $R h$. muhsamae est associé à $R h$. senegolensis, mais dans une région donnée les fréquences respectives de chacune de cès espèces ne sont jamais équivalentes; si $R h$. senegalensis domine numériquement au centre du Dahomey, partout ailleurs Rh. muhsomae semble être le plus commun ; dans certaines régions même $R$ h. senegalensis est rare ou localisé : Casamance, Guiné-Bissau, ovest de la Haute-Volta, ouest du Centre-Afrique (dans la mesure où la faune des tiques est connue avec plus de précision dans ces pays que dans des territoires anglophones comme le Ghana ou la Nigeria). Dans les savanes subéquatoriales guinéo-oubanguiennes et dans les mosaïques forêtsavane correspondantes, la présence de $R h$. senegalensis apparaît comme exclusive de $R h$. muhsamae de la Sierra-Leone au Cameroun ; par contre en Guiné-Bıssau et en Centre-Afrique, les deux espèces se rencontrent avec des abondances. variables à l'intérieur même de ces pays. Certains facteurs modifiant le microclimat interviennent vraisemblablement dans chaque zone de végétation ; il est possible que soient en cause
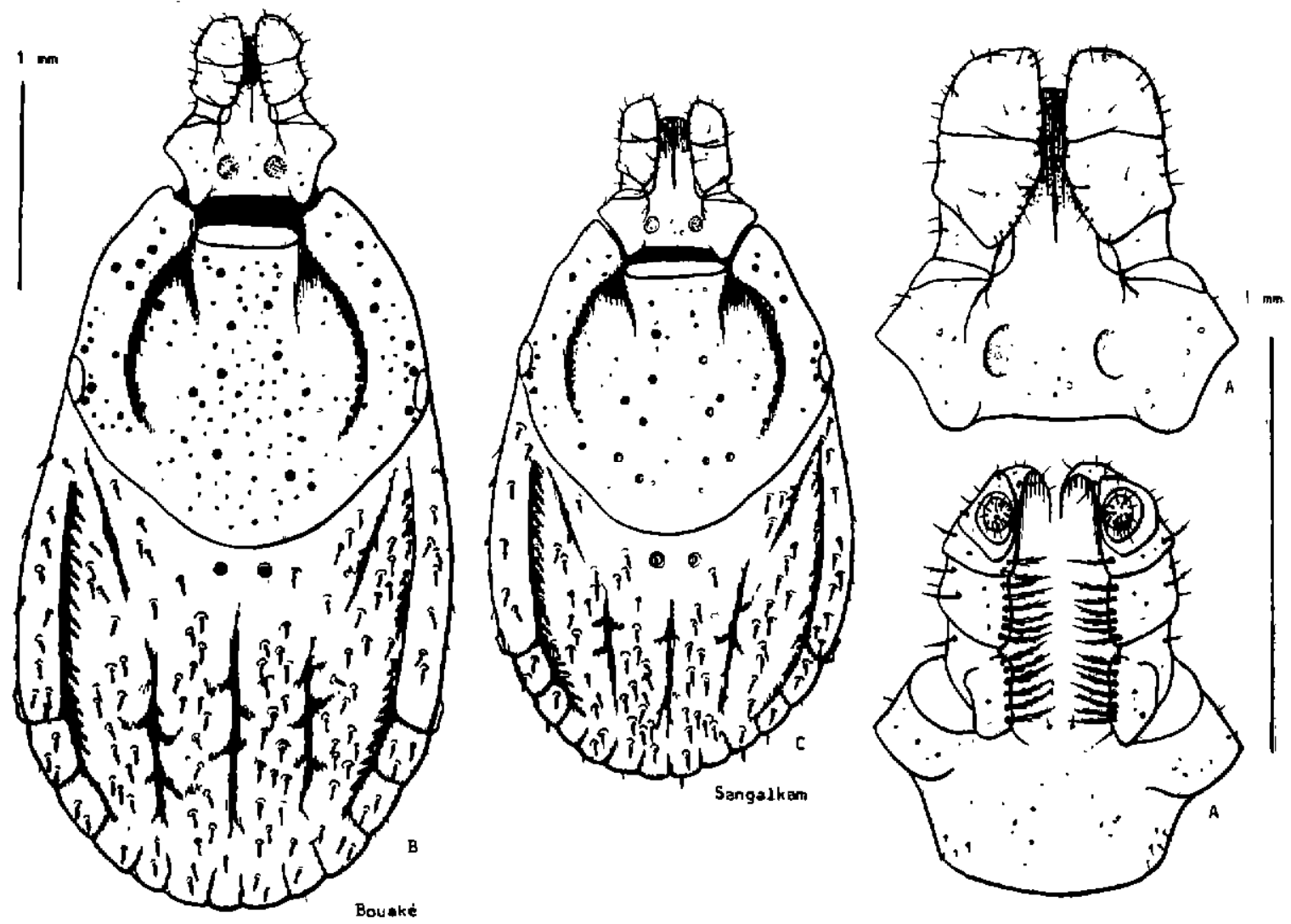

Fig. 13. - Rhipicephalus senegalensis, femelle: faces dorsales (exemplaires de Parakou ef Sangalkam) ; détails du capitulum (cf. fig. 12). 
les différences du nombre des mois pluvieux et l'existence de une ou deux saisons des pluies, ainsi que le caractère plus ou moins ouvert ou fermé des boisements de savanes. Ainsi Rh. muhsamae se présente comme mésophıle, tandis que $R h$. senegalensis est nettement hygrophile.

$R h$. senegalensis existe également dans les savanes équatoriales d'altitude ; il pourrait en être de même pour $R h$. muhsomae, éventuellement confondu avec $R h$. simus.

Le véritable Rh. simus est distribué en Afrique orientale et australe, dans les steppes xérophytes somaliennes ef masai, les savanes boisées tropicales éthiopiennes d̀ Oxytenanthera, peut-être les savanes boisées équatoriales d'altitude ( $c f$. ci-dessus), les savanes boisées subtropicales rhodésiennes et angoliennes et les formations particulières enclavées, les savanes tropicales australes, les prairies de montagne australe du low veld et du middle veld, partiellement du high veld, le maquis du Cap, les steppes xérophytes australes, les mosaïques côtières forêt-savane équatoriales orientales et les mosaïques forêtsavane tropicales australes.

Les références concernant la distribution de Rh. senegolensis correspondent pour la plupart aux références d'auteurs citées à propos de $R h$. muhsamae; pour complément d'information, il sera utile de se reporter aux données réunies à ce sujet par HOOGSTRAAL (1956: 732), aussi bien qu'en ce qui touche la distribution de $R h$. simus, compte tenu du fait que certaines réfé-
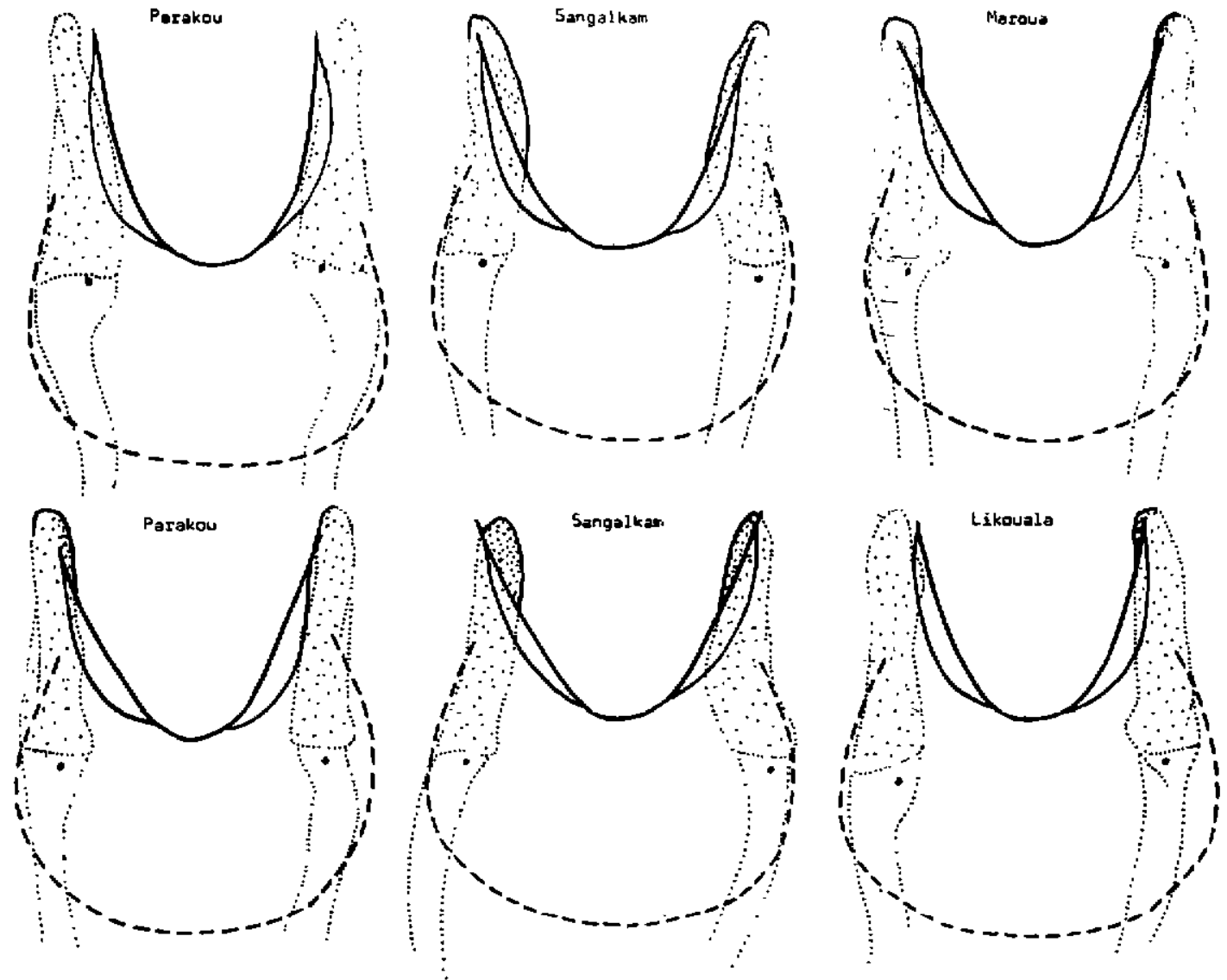

Fig. 14. - Rhipicephalus senegolensis, femelle; détail du gonopore (exemplaires de Sangalkam, Parakou, Maroua, Likouala). 
rences représentent en réalité Rh. muhsamoe': elles sont mentionnées dans ce cas dans le présent texte.

La terminologie des zones de végétation s'inspire presque entièrement de celle de la Carte de la végétation de l'Afrique, publiée par I'U. N. E. S. C. O. (Oxford, 1959).

\section{ROLE PATHOGENE DE RHIPICEPHALUS MUHSAMAE}

Tous les résultats obtenus à ce jour en ce qui concerne le véritable $R h$. simus, vis-à-vis de son pouvoir pathogène direct ou de son rôle vecteur de diverses affections humaines et animales, sont à confirmer à propos de $R h$. muhsamae.

Instifut d'élevage et de médecine vétérınaire des pays tropicaux. Alfort. Laborataire national de recherches vétérinaires Georges Curasson. Hann (Dakar).

\begin{abstract}
SUMMARY
Rhipicephalus muhsamae n. sp is notably distinct from Rh. simus, from the morphological point of view, by the structure of the female gonopore; and from $R$ h. senegalensis chiefly by the structure of the female gonopore and odanal plates in the male. Rh. simus, shows a typical distribution in austral and East Africa: Rh. muhsomae replaces it in West Africa, West of the Nile and in the Congo basin, up to Senegal; it is associated with tropical and sud-tropical savanna, sometimes sub-equatorial, as well as the steppes of southern Sahel, whereas $R h$. senegalensis, which is more hygrophile, has its normal, habitat in the subequatorial savanna, and less often in the sub-trapical; in West Africa the distribution of these two species occurs in parallel to the regions of different vegetation, where they mutually replace each other depending on the differences in the general or local climatic conditions.
\end{abstract}

\title{
RESUMEN
}

El Rhipicephalus muhsamae n. sp. se diferencia del Rh. simus, desde el punto de vista de su morfologia, por la estructura del gonoporo de la hembra ; y del Rh. senegalensis por el gonoporo y las placas adanales de los machos. El Rh. simus está distribuido de una manera tipica en Africa oriental y austral ; El Rh. muhsamae le reemplaza en Africa occidental al oeste del rio Nilo y en la cuenca del Congo, hasta el Senegal. Esta ligado con las sábanas tropicales y subtropicales, á veces con las subecuatoriales, asi como las estepas sursahelianas, mientras que el Rh. senegalensis, más higrófilo vive normalmente en las sábanas subecuatoriales, menos frecuentemente en las subtropicales; en el oeste de Africa la repartición de las dos especies es paralela a las zonas de vegetación, en las cuales se reemplazan mutuamente en función de las diferencias climáticas generales o locales.

\section{BIBLIOGRAPHIE}

AUBREVILLE (A), DUVIGNEAUD (P.), HOYLE (A. C.), KEAY: (R. W. J.), MENDONCA (F. A.) \& PICHI-SERMOLLI (R. E. G.) (1959):-
Vegetation map of Africa south of the tropic of Cancer. Carte de la végétation de l'Afrique au sud du tropique du Cancer. 
Explanatory notes by KEAY (R. W. J.) ; traduction d'AUBREVILLE (A.). U.N. E.S. C. O. (Oxford Univ. Press) : 1-24, 1 carte. HOOGSTRAAL $\left(H_{\text {. }}\right)$ (1956). - African Ixodoidea I. Ticks of the Sudan. Res. Rep. NM 005 050.29.07. Washington (U. S. Govt. Print. Office), 0-390 $800: 1100 \mathrm{pp}$.

HOOGSTRAAL (H.) \& THEILER (G.) (1959). Ticks (Ixodoidea, Ixodidae) parasitizing lower primates in Africa, Zanzibar and Madagescar. J. Parasit., 45 (2) : 217-222.

LAMONTELLERIE (M.) (1960). - Tiques (Acarina, Ixodoidea) du cercle de Sikasso (République soudanaise), Bull. Soc. Path. exot., 53 (4) : 751-755.

MOREL (P. C.) (1956). - Tiques d'animaux sauvages. Mém. Inst. fr. Afr. noire, 48 (Le parc national du Niokolo-Koba, Sénégal, l) : $229-232$.

MOREL (P. C.) (1958). - Les tiques des animaux domestiques de l'Afrique-Occidentale française Rev. Elev. Méd. vét. Pays trop., 11 (2) : 153-189.

MOREL (P. G.) (1961). - Tiques (Acarina, Ixodoidea). Deuxième note. Mém. Inst. fr. Afr. norre, 62 (Le parc national du NiokoloKoba, II) : 83-90.

MOREL (P. C.) \& FINELLE (P.) (1961). - Les tiques des animaux domestiques du CentreAfrique. Rev. Elev. Méd. vét. Pays trop., 14 (2) : 191-197.

MOREL (P. C.) \& GRABER (M.) (1961), - Les tiques des animaux domestiques du Tchad. Rev. Elev. Méd. vét. Pays trop., 14 (2) : 199-203.

MOREL (P. C.) \& MAGIMEL (J.) (1959). - Les tiques des animaux domestiques de la région de Fort-Lamy (Tchad) ef Fort-Foureau ( $\mathrm{Ca}$ meroun). Rev. Elev. Méd. vét. Pays trop., 12 (1) : 53-57.

MOREL (P. C.) \& MOUCHET (J.) (1958). - Les tiques du Cameroun (Ixodidae et Arguasidae). Ann. Porasit. hum. comp., 33 (1-2) : 69-111.

MOREL (P. C.) \& MOUCHET (J.) (1965). - Les tiques du Cameroun (Acariens, Ixodoidea).
Deuxième note. Ann. Parasit. hum. comp., 39 (sous presse).

NEUMANN (L. G.) (1902). - Notes sur les Ixodidés. I. Arch. Parasit., 6(1) : 109-128.

NEUMANN (L. G.) (1902). - Note sur une collection d'ectoparasites recueillis en Abyssinie par $M$. le baron $C$. von Erlanger. Arch. Parasit., 6 (2) : 293-294.

ROUBAUD (E.) \& VAN SACEGHEM (R.) (1916). - Observations sur quelques insectes ef acariens parasites du bétail du Congo-Belge. Bull. Soc. Poth. exot., 9 (10) : 763-767.

SCHWETZ (J.) (1927). - Contribution à l'étude des Ixodidae (tiques) du Congo-Belge (d'après la collection du Musée de TervuerenBruxelles) (troisième note). Rev. Zool. afr., 15 (1) $: 81-92$.

SCHWETZ (J.) (1932), -- Notes géographiques d'entomologie médicale sur la ProvinceOrientale (Congo-Belge) (tiques, tabanides, maringouins). Ann. Soc. belge Méd. trop. 12 (4) : 549-555.

ROUSSELOT (R.) (1951). - Ixodes de l'Afrique noire. Bull. Soc. Poth. exot., 44 (5-6) : 307-309.

ROUSSELOT (R.) (1953). - Notes de parasitologie tropicale. II. Ixodes. Paris (Vigot Edit.) : 1-152.

TENDEIRO (J.) (1946), - Ixodideos da Guiné Portuguesa : Rhipicephalus simus Koch, 1844 e Rh. sanguineus (Latreille, 1806). Bol. cult. Gunné port. 1 (3) : 397-423.

TENDEIRO (J.) (1948). - Subsidios para o conhecimiento da fauna parasitologica da Guiné, Bol. cult. Guiné port., 3 (11) : 639-738.

TENDEIRO (J.) (1956). - Notas sobre o Rhipicephalus simus simus C. L. Koch, $1844 \mathrm{e}$ - Rhipicephalus simus senegalensis C. L. Koch, 1844 na Guiné Portuguesa. Bol. cult. Guné port., 11 (42) : 99-109.

TENDEIRO (J.) (1959). - Sur quelques ixodidés du Mozambique ef de la Guinée Portugaise. I. Bol. cult. Guiné port., 14 (53) : 21-95. 
THEILER (G.) \& ROBINSON (B. N.) (1954). Tick survey VIII. Check-list if ticks recorded from the Belgian Congo and RuandaUrundi, from Angola and from Northern Rhodesia, Onderst. J. vet. Res., 26 (3) : 447461.

UNSWORTH (K.) (1952). - The ixodid parasites of cattle in Nigeria. Ann. trop. Med. Parasit., $46(4): 331-336$.

VAN VAERENBERGH (R.) (1954). - Notes relatives à quelques déterminations de tiques du Ruanda-Urundi et du Congo-Belge. Bull. Ann. Soc. entom. Belgique., 90 (7-8) : 222-226.

VASSILIADES (G.) (1965). - Contribution à la connaissance de la tique africaine Rhipicephalus senegalensis Koch, 1844 (Acariens, Ixodoidea). Ann. Fac. Sci. Dakar, 14 (sous presse).

ZUMPT (F.) (1943) - - Vorstudie zu einer Revision der Gattung Rhlpicephalus Koch. VII. Rhipicephalus simus Koch und verwandte Arten. Ztschr. Parasitenk., 13 (1) : 1-24. 